IJMMS 29:10 (2002) 609-611

PII. S0161171202007974

http://ijmms.hindawi.com

(c) Hindawi Publishing Corp.

\title{
A GENERALIZATION RELATED TO SCHRÖDINGER OPERATORS WITH A SINGULAR POTENTIAL
}

\author{
TOKA DIAGANA
}

Received 30 July 2001

The purpose of this note is to generalize a result related to the Schrödinger operator $L=-\Delta+Q$, where $Q$ is a singular potential. Indeed, we show that $D(L)=\{0\}$ in $L^{2}\left(\mathbb{R}^{d}\right)$ for $d \geq 4$. This fact answers to an open question that we formulated.

2000 Mathematics Subject Classification: 35J05, 47B25, 47B44.

1. Introduction. Let $L$ be the operator of Schrödinger, defined in $L^{2}\left(\mathbb{R}^{d}\right)$ as $L=$ $A+B$, where

$$
\begin{gathered}
A \phi=-\Delta \phi, \quad D(A)=H^{2}\left(\mathbb{R}^{d}\right), \\
B \phi=Q \phi, \quad D(B)=\left\{\phi \in L^{2}\left(\mathbb{R}^{d}\right): Q \phi \in L^{2}\left(\mathbb{R}^{d}\right)\right\} .
\end{gathered}
$$

We suppose that the potential $Q$, verifies the following conditions, see, for example, [1],

$$
Q>0, \quad Q \in L^{1}\left(\mathbb{R}^{d}\right), \quad Q \notin L_{\mathrm{loc}}^{2}\left(\mathbb{R}^{d}\right) .
$$

Under these conditions we show that $D(L)=\{0\}$, for $d \geq 4$, this fact extends the author's result (the case where $d \leq 3$, see the details in [1]). For that we use approximations of functions of $H^{2}\left(\mathbb{R}^{d}\right)$ (when $d \geq 4$ ) by continuous functions in connection with BMO space (where BMO $\left(\mathbb{R}^{d}\right)$ is the space of functions of Bounded Mean Oscillation), see [2]. Let $\phi \in L^{2}\left(\mathbb{R}^{d}\right)$, we denote by $I_{\alpha}$ the operator defined by

$$
I_{\alpha} \phi=(-\Delta)^{-\alpha / 2} \phi=\sqrt{(-\Delta)}^{(-\alpha)} \phi .
$$

Thus, we know that

$$
\left\|I_{\alpha} \phi\right\|_{L^{q\left(\mathbb{R}^{d}\right)}} \leq C_{p, q, d}\|\phi\|_{L^{p}\left(\mathbb{R}^{d}\right)}, \quad \text { if } \frac{1}{q}=\frac{1}{p}-\frac{\alpha}{d} \text { with } \frac{1}{p}>\frac{\alpha}{d} .
$$

In the case where $1 / p=\alpha / d, \alpha=p=2$, and $d=4$, then

$$
\left\|I_{2} \phi\right\|_{\mathrm{BMO}} \leq C\|\phi\|_{2} \text {. }
$$

We also have

$$
I_{2}\left(C_{0}^{\infty}\left(\mathbb{R}^{d}\right)\right) \subseteq \mathrm{VMO}
$$

where $\operatorname{VMO}\left(\mathbb{R}^{d}\right)$ is the space of functions of Vanishing Mean Oscillation. We can find details in [2]. 
2. Generalization. Let $H$ be a Hilbert space given by $H=L^{2}\left(\mathbb{R}^{d}\right)$, thus we have the following proposition.

Proposition 2.1. Under the previous hypotheses on the singular potential $Q$ and if $d \geq 4$, then

$$
D(L)=\{0\} .
$$

Proof. Let $u \in D(A) \cap D(B)$, suppose that $u \neq 0$, then there exists an open subset $\Omega$ of $\mathbb{R}^{d}$ such that $|u(x)|>a$, for all $x \in \Omega \subseteq \operatorname{supp} u$. Let $\Omega^{\prime} \subseteq \Omega$, be a compact subset of $\Omega$.

STEP 1. When $d \leq 3$, done in [1].

STEP 2. Suppose $d=4$. Then there exists $\left(u_{k}\right) \in C_{0}^{\infty}\left(\mathbb{R}^{4}\right)$ such that $u_{k}$ converges to $u$ into $H^{2}\left(\mathbb{R}^{4}\right)$, thus, we can write $u_{k}=I_{2} v_{k}$ and $u=I_{2} v$ and $v \in L^{2}\left(\mathbb{R}^{4}\right)$. It follows that

$$
\left\|u_{k}-u\right\|_{\mathrm{BMO}} \leq C\left\|v_{k}-v\right\|_{2} \rightarrow 0
$$

because $v_{k}$ converges to $v$ into $L^{2}\left(\mathbb{R}^{4}\right)$, thus $u_{k}$ converges to $u$ into BMO. Consider $u_{k}$ and $u$ as functions defined on $\Omega^{\prime}$, then $|Q|_{\mid \Omega^{\prime}}=\left(\left|Q u_{k}\right| /|u|_{k}\right)_{\mid \Omega^{\prime}}$, on passing to the limit in BMO and by the fact that $B$ is a closed operator. It follows that $Q \in L^{2}\left(\Omega^{\prime}\right)$, that is impossible according to the hypothesis on the potential, $Q \notin L_{\mathrm{loc}}^{2}\left(\mathbb{R}^{4}\right)$. And then, we conclude that $u=0$.

STEP 3. Suppose $d>4$, and write $u_{k}=I_{2} V_{k}$ and $u=I_{2} v$ where $v_{k}$ converges to $v$ into $L^{2}\left(\mathbb{R}^{d}\right)$. Thus, $\alpha=p=2$ and $1 / q=1 / 2-2 / d$ where $d>4$, therefore,

$$
\left\|u_{k}-u\right\|_{q}=\left\|I_{2} v_{k}-I_{2} v\right\|_{q} \leq C\left\|v_{k}-v\right\|_{2}
$$

then $u_{k}$ converges to $u$ into $L^{q}\left(\mathbb{R}^{d}\right)$. We also write, $Q=Q u_{k} / u_{k}$, and consider this function on $\Omega^{\prime}$ and by passing to the limit into $L^{q}\left(\mathbb{R}^{d}\right)$, we get a contradiction.

Conclusion. The domain of the algebraic sum of $A$ and $B$ is always zero, that is, $D(A) \cap D(B)=\{0\}$, without restriction on $d$.

REMARKS. The dimensional $d$ of $\mathbb{R}^{d}$ has no impact on the sum form of $A$ and $B$, $(-\Delta \oplus Q)$. This operator is always defined and verifies Kato's condition and is given as

$$
\begin{gathered}
D((-\Delta \oplus Q))=\left\{u \in H^{1}\left(\mathbb{R}^{d}\right): Q|u|^{2} \in L^{1}\left(\mathbb{R}^{d}\right),-\Delta u+Q u \in L^{2}\left(\mathbb{R}^{d}\right)\right\}, \\
(-\Delta \oplus Q) u=-\Delta u+Q u
\end{gathered}
$$

therefore, Kato's condition is satisfied, that is,

$$
D(\sqrt{(-\Delta \oplus Q)})=D(\sqrt{-\Delta}) \cap D(\sqrt{Q})=D(\sqrt{(-\Delta \oplus Q) *}) .
$$

The example of singular potential given in [1] is always valid for all $d$,

$$
Q(x)=\sum_{k=0}^{+\infty} \frac{G\left(x-\alpha_{k}\right)}{k^{2}},
$$


where $G$ is a function defined on the compact subset $\Omega$ of $\mathbb{R}^{d}$ and verifying

$$
G>0, \quad G \in L^{1}(\Omega), \quad G \notin L^{2}(\Omega), \quad G=0 \quad \text { on } \mathbb{R}^{d}-\Omega,
$$

where $\alpha_{k}=\left(\alpha_{k}^{1}, \alpha_{k}^{2}, \ldots, \alpha_{k}^{d}\right) \in Q^{d}$ is a rational sequence.

Acknowledgment. I want to express my thanks to Professor Steve Hofmann, from University of Missouri at Columbia about the discussions we had had on the point solved in this note.

\section{REFERENCES}

[1] T. Diagana, Schrödinger operators with a singular potential, Int. J. Math. Math. Sci. 29 (2002), no. 6, 371-373.

[2] E. M. Stein, Singular Integrals and Differentiability Properties of Functions, Princeton Mathematical Series, no. 30, Princeton University Press, New Jersey, 1970.

Toka Diagana: Department of Mathematics, Howard University, 2441 Sixth Street, NW, WASHINGTON, DC 20059, USA

E-mail address: tdi agana@howard.edu 


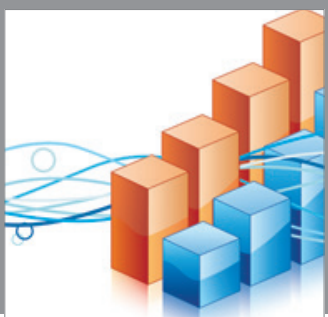

Advances in

Operations Research

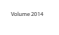

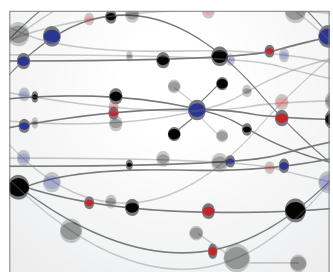

\section{The Scientific} World Journal
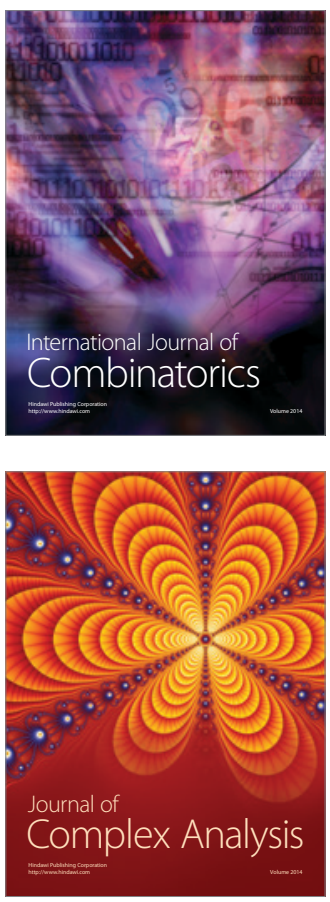

International Journal of

Mathematics and

Mathematical

Sciences
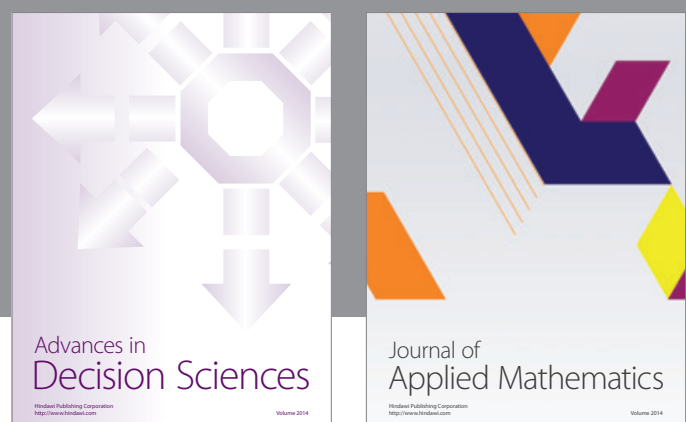

Journal of

Applied Mathematics
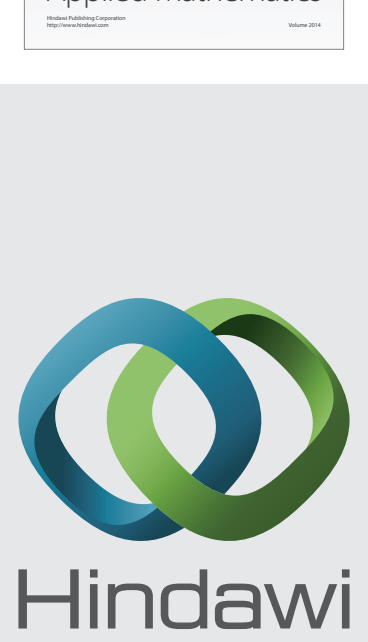

Submit your manuscripts at http://www.hindawi.com
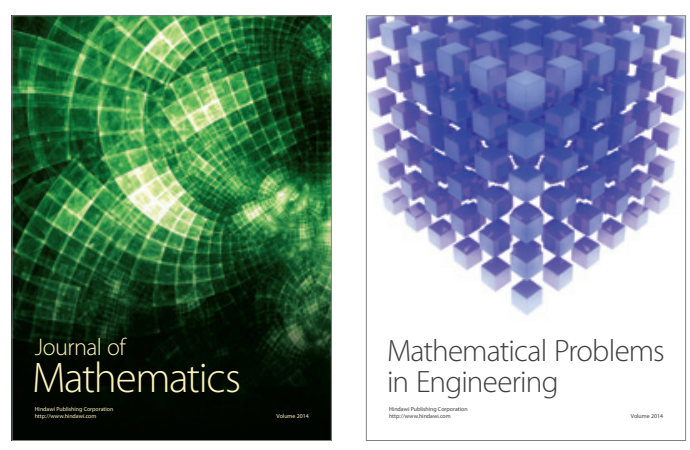

Mathematical Problems in Engineering
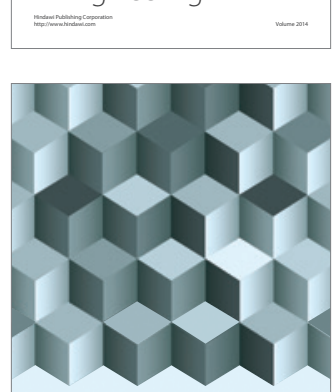

Journal of

Function Spaces
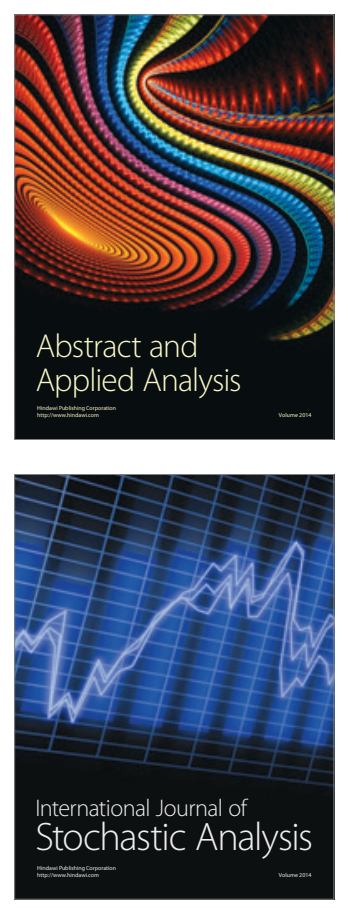

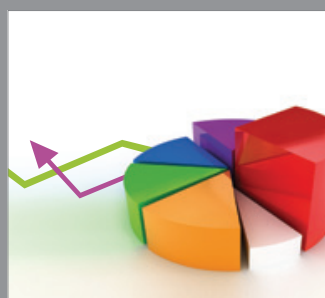

ournal of

Probability and Statistics

Promensencen
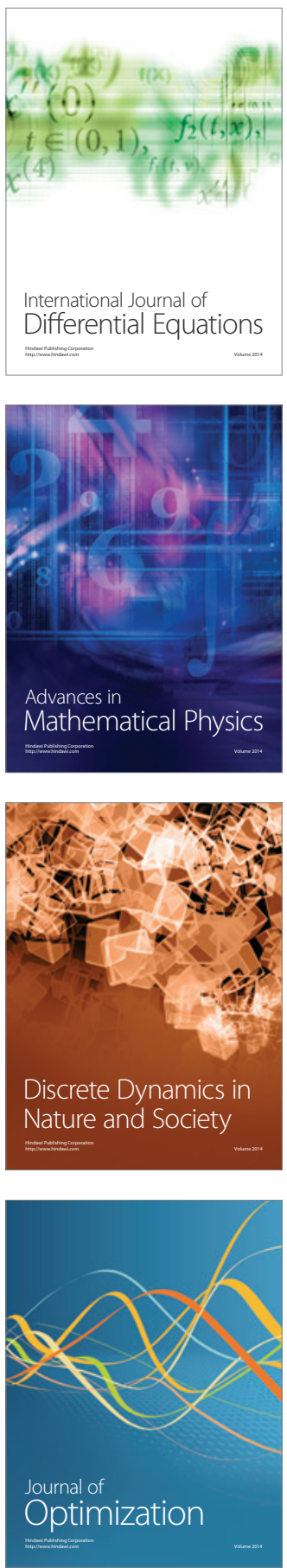